\title{
A New Sensitive Fluorimetric Method for the Determination of Heptaminol and Mexiletine in Pharmaceuticals
}

\author{
Sobhy M. EI-AdI \\ Department of Pharmaceutical Chemistry, Faculty of Pharmacy \\ University of Zagazig, Zagazig, Egypt
}

\begin{abstract}
:
An accurate and very sensitive spectrofluorimetric method is proposed for the estimation of heptaminol (HP) and mexiletine (MX) in their pure forms and in pharmaceutical formulations. The method is based on the reaction of these drugs with the strong fluorigenic reagent [9,10-Dimethoxy-2-anthracenesulfonic acid sodium salt (DAS)] at $\mathrm{pH}$ (2.5). The formed complexes are extracted into chloroform and measured spectrofluorimetrically. The maximum wavelengths of excitation and emission spectra of heptaminol complex are 381 and $441 \mathrm{~nm}$, respectively. While those of mexiletine complex are 385 and $450 \mathrm{~nm}$, respectively. Heptaminol can be determined in the concentration range of $0.016-0.144 \mu \mathrm{g} \mathrm{ml}^{-1}$ and that of mexiletine in the range of $0.024-0.176 \mu \mathrm{g} \mathrm{ml}^{-1}$, when extracted from the solutions into chloroform. Different parameters affecting the reaction conditions were thoroughly studied. No interference was observed in the presence of common pharmaceutical excipients. The proposed method has been successfully applied to the analysis of commercial pharmaceutical formulations and the results have been statistically compared with those obtained by the reference methods.
\end{abstract}

Keywords: Heptaminol; Mexiletine; 9,10-Dimethoxy-2-anthracenesulfonic acid sodium salt; Fluorimetry; Pharmaceuticals.

\section{Introduction:}

Heptaminol (HP) and Mexiletine (MX) are cardiovascular drugs containing an aliphatic primary amino group in their structures. Chemically, HP is (6-amino-2methyl-2-heptanol hydrochloride) and medicinally used as cardiac \& circulatory stimulant. While MX is (1-methyl-2- (2,6-xylyloxy) ethylamine hydrochloride) and used as an antiarrhythmic agent. $H P$ is non official drug and only one spectrophotometric \& spectrofluorimetric method, based on Hantzsch reaction, was found in the literature for its determination ${ }^{(1)}$. The other quantitation methods of HP 
and MX are mainly based on chromatographic techniques ${ }^{(2-7)}$. MX was also determined potentiometrically ${ }^{(8,9)}$ or by using the capillary electrophoresis technique ${ }^{(10,1)}$. The USP (XXIV) $)^{(12)}$ describes a reversed phase HPLC method for MX assay, while the BP $(1998)^{(13)}$ describes a non aqueous titration method for its assay.

Due to HP and MX are very weakly UV-absorbing drugs, where MX has the value of $\mathrm{A}(1 \%, 1 \mathrm{~cm})=14$ in aqueous acid ${ }^{(14)}$, thus their determinations by direct $\mathrm{UV}$ measurement are unreliable. Therefore, it was felt useful to develop a selective and sensitive method for estimation of HP and MX in their pharmaceuticals. In this article, Heptaminol and Mexiletine are determined in a pure form and in different pharmaceutical products by the interaction with 9, 10-dimethoxy-2-anthracenesulfonic acid sodium salt in presence of buffer at $\mathrm{pH}(2.5)$. The resulted products are extracted into chloroform and measured spectrofluorimetrically with high sensitivity.

\section{Experimental:}

Instruments:

1-RF-1501 Spectrofluorometer, Shimadzu (Japan), with $1 \mathrm{~cm}$ quartz cuvettes and interfaced with a computer system.

2- Chemocadet pH-meter, model 5984-50, connected to a glass calomel electrode (USA).

\section{Materials and Reagents:}

9,10-Dimethoxy-2-anthracenesulfonic acid sodium salt (DAS); Fluka Chemika (Buchs, Switzerland); was prepared as $6 \times 10^{-4} \mathrm{M}$ solution by dissolving $20.4 \mathrm{mg}$ in $100 \mathrm{ml}$ of bidistilled water. Chloride buffer and acetate buffer solutions $\mathrm{pH}$ (1.2$5.5)^{(15)}$; the $\mathrm{pH}$ was adjusted using the $\mathrm{pH}$-meter. Anhydrous sodium sulfate. Chloroform (Prolabo). Heptaminol $\mathrm{HCl}$ (Pharco Pharm, Alexandria, Egypt). Mexiletine $\mathrm{HCl}$ (Boehringer Ingelheim International $\mathrm{GmbH}$, Germany). Corasore tablets (Amoun Pharm, Cairo) labeled to contain $150 \mathrm{mg}$ of heptaminol $\mathrm{HCl}$ per each tablet. Mexitil capsules (Boehringer Ingelheim) labeled to contain $200 \mathrm{mg}$ of mexiletine $\mathrm{HCl}$ per each capsule.

\section{Procedures:}

\section{Standard solution preparations:}

$\mathrm{HP}$ and MX were prepared as a stock solution of $0.5 \mathrm{mg} \cdot \mathrm{ml}^{-1}$ by dissolving 50 $\mathrm{mg}$ of $\mathrm{HP}$ and $\mathrm{MX}$ hydrochlorides in a $100 \mathrm{ml}$ volumetric flask with completion to the mark with de-ionized water. Exactly, $1 \mathrm{ml}$ of each $\mathrm{HP}$ and $\mathrm{MX}$ stock solution was transferred into a $250-\mathrm{ml}$ volumetric flask and the volume was completed to the mark 
with de-ionized water. The resulted working standard solutions were obtained to contain $2 \mu \mathrm{g} \cdot \mathrm{ml}^{-1}$ of each HP and MX.

\section{Construction of the calibration graphs:}

Into a dry series of $50 \mathrm{ml}$ separatory funnels, different aliquots of the working standard solutions equivalent to 0.4-3.6 $\mu \mathrm{g}$ of $\mathrm{HP}$ and 0.6-4.4 $\mu \mathrm{g}$ of $\mathrm{MX}$ were transferred. Then, $3 \mathrm{ml}$ of the acetate buffer solution of $\mathrm{pH} 2.5$ followed by $2 \mathrm{ml}$ of DAS reagent were added. The formed complex was extracted with three- $7 \mathrm{ml}$ quantities of chloroform by shaking for 5 minutes. The combined chloroformic extracts were filtered through anhydrous sodium sulfate into a $25 \mathrm{ml}$ volumetric flasks and completed to volume with the same solvent. The fluorescence intensity was then measured at $441 \mathrm{~nm}$ for HP (after excitation at $381 \mathrm{~nm}$ ) and at $450 \mathrm{~nm}$ for MX (after excitation at $385 \mathrm{~nm}$ ) against the corresponding reagent blank.

\section{Determination of pharmaceutical formulations:}

An accurately weighed quantity of either tablet's powder (Corasore tablets containing HP-HCl) or capsule's powder (Mexitil capsules containing $\mathrm{MX}-\mathrm{HCl}$ ) equivalent to $50 \mathrm{mg}$ of each drug was quantitatively transferred to $100-\mathrm{ml}$ volumetric flask. Approximately, $40 \mathrm{ml}$ of de-ionized water was added and the content was well shaken for $10 \mathrm{~min}$., completed to the volume and filtered. Exactly, $1 \mathrm{ml}$ of the filtrate of each drug was transferred into a $250 \mathrm{ml}$ volumetric flask and the volume was completed to the mark with de-ionized water. An aliquot portions of the final solution were transferred into a dry series of $50-\mathrm{ml}$ separatory funnels and the procedure was then completed as mentioned before.

\section{Results and Discussion:}

Since HP and MX contain an aliphatic primary amino group in their chemical structures, they can be readily protonated at a suitable $\mathrm{pH}$ medium. The ion-pair extraction of analytes with the counter ion aromatic sulfonic acids such as 9,10dimethoxyanthracene-2-sulfonate (DAS) was producing a strong fluorescence. Based on these principles, certain amines were detected on a high-performance liquid chromatographic columns with high sensitivity ${ }^{(16-19)}$. Such properties were utilized for the quantitative determination of $\mathrm{HP}$ and $\mathrm{MX}$ spectrofluorimetrically in pure and dosage forms. The cited drugs react instantaneously with DAS reagent in the presence of buffer at $\mathrm{pH} 2.5$ to form a highly fluorescent complexes. Thus, the formed complexes were extracted into chloroform and measured fluorimetrically at $\lambda 441 \mathrm{~nm}$ 
when excitation was at $\lambda 381 \mathrm{~nm}$ (in case of HP-complex); and measured at $\lambda 450 \mathrm{~nm}$ when excitation was at $\lambda 385 \mathrm{~nm}$ (in case of MX-complex), Figs. $(1,2)$.

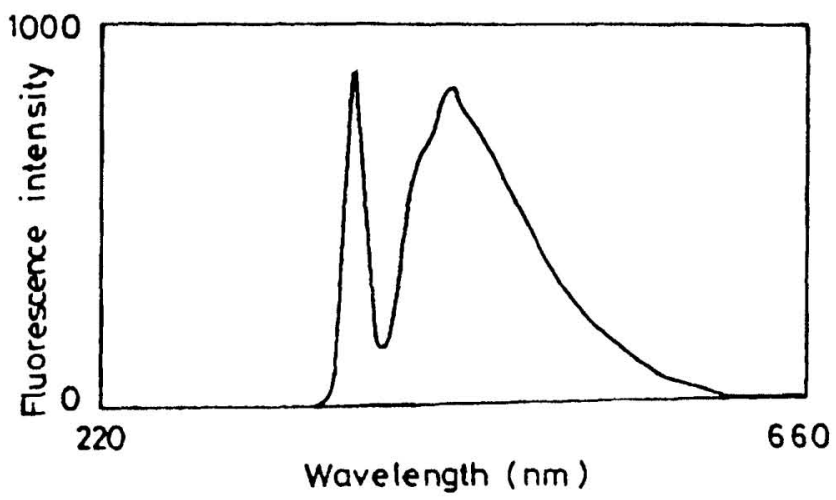

Fig. (1): Fluorescence excitation and emission spectra of Heptaminol $\left(0.128 \mu \mathrm{g} \cdot \mathrm{ml}^{-1}\right)$ complex with DAS reagent.

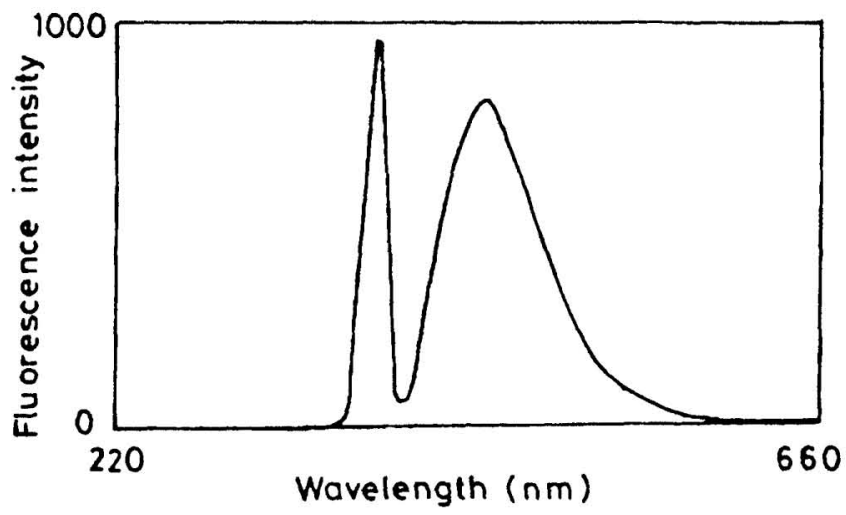

Fig. (2): Fluorescence excitation and emission spectra of Mexiletine $\left(0.152 \mu \mathrm{g} \cdot \mathrm{ml}^{-1}\right)$ complex with DAS reagent.

Various parameters affecting the reaction process were studied. It was found that the reaction is a strongly $\mathrm{pH}$-dependant. Thus, the $\mathrm{pH}$ of the reaction medium was varied to observe the relative fluorescence under the reaction conditions. The most suitable $\mathrm{pH}$ for the formed complexes was found to be in the range (2-3), where the highest possible concentration of analytes was present in this range. Above $\mathrm{pH} 4$, larger proportion of drugs become unionized. Maximum fluorescence was obtained upon using $3 \mathrm{ml}$ of acetate buffer solution of $\mathrm{pH} 2.5$. 
The influence of the reagent concentration, reaction time and reaction temperature, selected for the proposed method, were also studied. The optimum concentration of DAS-reagent was chosen to be $6 \times 10^{-4} \mathrm{M}$ solution, where $2 \mathrm{ml}$ of this concentration gave the maximum fluorescence intensity. Regarding the reaction time and temperature, it was found that shaking for about $5 \mathrm{~min}$ at room temperature $\left(25^{\circ} \mathrm{C}\right)$ was enough to develop the optimal fluorescence intensity. The intensity of the produced fluorescence stayed constant for up to two hours after extraction of the samples.

Several organic solvents were examined concerning the extraction efficiency and the fluorescence intensity, such as chlorobenzene, toluene, chloroform and methylene chloride. It was found that chloroform is the best solvent with respect to the excellent extractibility, low blank value and the higher sensitivity. The continuous variation method of Job's was applied in order to study the composition of complexes and the reaction's stoichiometry. Results revealed a 1:1 complexation ratio under the optimal conditions attained for the reaction between the drug (HP or MX) and DASreagent. This finding was anticipated due to the presence of a single basic moiety in their structures.

Applying the optimal experimental conditions attained, the standard calibration graphs were developed for the proposed fluorimetric reaction. A linear correlation was obtained between the fluorescence intensity and the concentration in the range of 16$144 \mathrm{ng} \cdot \mathrm{ml}^{-1}$ and 24-176 ng.ml ${ }^{-1}$ for each of $\mathrm{HP}$ and $\mathrm{MX}$, respectively. The concentration of different unknown samples can be calculated from such calibration graphs or by using the corresponding regression equations:

$\mathrm{F}_{441 \mathrm{~nm}}=0.874+6.45 \mathrm{C}$
$\mathrm{F}_{450 \mathrm{~nm}}=0.492+5.50 \mathrm{C}$
$\ldots \ldots \ldots \ldots \ldots \ldots \ldots \ldots \ldots \ldots \ldots \ldots \ldots \ldots \ldots \ldots \ldots \ldots \ldots \ldots \ldots \ldots \ldots \ldots \ldots \ldots \ldots \ldots \ldots \ldots$

Where $(\mathrm{C})$ is the concentration of drug in $\mathrm{ng} \cdot \mathrm{ml}^{-1}$ and $(\mathrm{F})$ is the fluorescence intensity at the specified $\lambda(\mathrm{nm})$ of emission and excitation. The correlation coefficient ( $\mathrm{r}$ ) values were found to be in the order of 0.9995 and 0.9997 for HP and MX, respectively.

Regression analysis of the obtained results were presented using the method of least squares to calculate the intercept, slope and correlation coefficient ${ }^{(20)}$. Five separate determinations were carried out using different concentrations of both HP and MX to test the reproducibility. The relative standard deviations were found to be less than $2 \%$, indicating excellent reproducibility of the proposed method. The small values of intercepts (a) and high values of correlation coefficient ( $r$ ) validated the linearity of 
the calibration graphs and the obedience to Beer's law. The limits of detection was derived from the standard deviation of the blank measurements and from the slope of the calibration graph, found to be 3.4 and $4.8 \mathrm{ng} \cdot \mathrm{ml}^{-1}$ for HP and MX, respectively. This limit is adequate for clinical analysis of both drugs in the biological fluids.

Different batches of HP and MX pharmaceutical preparations were analyzed for their contents by the proposed method and satisfactory results were obtained. Moreover, the validity of the suggested procedure was assessed by applying the standard addition technique and the results obtained were represented in (Tables 1,2). To prove the applicability of the proposed method and the reproducibility of the obtained results of both HP and MX in their dosage forms, the results were compared statistically with those obtained by the reference spectrofluorimetric method based on Hantzsch reaction ${ }^{(1)}$. From (Tables 1,2), it is clear that the calculated t- and F-values were less than the theoretical ones. Thus concludes that there is no significant difference between the two methods with respect to accuracy and precision ${ }^{(21)}$.

Table (1): Quantitative determination of Heptaminol (in Corasore tablets) using the proposed method compared with the reference spectrofluorimetric method $^{(1)}$.

\begin{tabular}{|l|c|c|}
\hline \multicolumn{1}{|c|}{ Parameters } & Reference method & Proposed method \\
\hline Mean recovery* & 99.24 & 99.60 \\
Variance & 0.982 & 0.845 \\
SD & 0.991 & 0.919 \\
SE & 0.444 & 0.412 \\
RSD (\%) & 0.998 & 0.923 \\
t-test & & $0.59(2.31)^{* *}$ \\
F-test & & $1.16(6.39)^{* *}$ \\
\hline
\end{tabular}

Table (2): Quantitative determination of Mexiletine (in Mexitil capsules) using the proposed method compared with the reference spectrofluorimetric method $^{(1)}$.

\begin{tabular}{|l|c|c|}
\hline \multicolumn{1}{|c|}{ Parameters } & Reference method & Proposed method \\
\hline Mean recovery* & 100.80 & 100.58 \\
Variance & 1.075 & 0.86 \\
SD & 1.037 & 0.927 \\
SE & 0.465 & 0.416 \\
RSD (\%) & 1.030 & 0.921 \\
t-test & & $0.36(2.31)^{* *}$ \\
F-test & & $1.25(6.39)^{* *}$ \\
\hline
\end{tabular}

* Number of experiments $=5$; where each result is the average of triplicate measurements.

** Figures in parenthesis are the corresponding tabulated values for $t-$ and $F$-tests $($ at $P=0.05)$. 
The proposed method was found to be selective for the determination of either HP or MX. There was no evidence of interference from the common excipients in the examined pharmaceutical preparations. The effect of incorporated additives such as starch, lactose, avicel and magnesium stearate was experimentally studied showing no fluorescence or interference with the assay. The similarity of the regression equations of authentic samples of the two drugs to that of their dosage forms, as well as the high correlation coefficient values indicate the non interference in the fluorescence emission at the chosen wavelengths.

\section{Comparison with the other analytical methods:}

Few articles have appeared in the literature for the determination of both HP and MX in pharmaceuticals. The techniques used in this connection include mainly chromatographic methods (GLC, HPLC). The GLC method requires oftently preparation of the derivatives with a tedious extraction steps. The HPLC method requires sophisticated instrument and expensive reagents. The official BP describes a non aqueous titration method for assay of $\mathrm{MX}$ in bulk powder ${ }^{(13)}$, that requires at least $150 \mathrm{mg}$ of drug for its determination. While the BP describes a direct UV measurement at $\lambda 260 \mathrm{~nm}$ for assay of MX in pharmaceutical preparations ${ }^{(13)}$, that has distinct disadvantages of very low sensitivity and suffers from interference at that wavelength.

Since HP is non official drug, and both of HP and MX are very weakly UV absorbing compounds, the need to find sensitive efficient analysis method for the two drugs is of great importance. Spectrofluorimetry is a useful and powerful technique for this purpose because of high sensitivity, rapidity, simplicity and low cost. The sensitivity of the fluorimetric assay is well known and this approach was chosen for investigation. It was considered desirable to substitute a simple and sensitive assay procedure that would be suitable for all of these analyses. Therefore, there has been an increased interest in developing an accurate analytical methods valid for the quantification of these two drugs in biological and pharmaceutical samples. The proposed method is the most sensitive one compared to the other reported methods for assay of both HP and MX.

\section{Conclusion:}

A sensitive spectrofluorimetric procedure has been developed to provide simple, rapid and accurate method for estimation of HP and MX in bulk authentic 
samples and in pharmaceutical formulations. The method has been fully validated and is linear down to $16 \mathrm{ng}^{\mathrm{ml}}{ }^{-1}$ with good accuracy and precision. A comparative examination of batches of HP-tablets and MX-capsules using the proposed and existing techniques demonstrated equivalent results with respect to accuracy and precision. But the proposed method is significantly faster and more sensitive than the current procedures. The presence of excipients in the commercial formulations did not affect the accuracy of the method. It is noteworthy to mention that the proposed method is regarded as being superior to the official BP method because it can be used for determination of the two drugs in trace amounts. Therefore, it could be easily used in quality control laboratories for the analysis of both HP and MX in bulk materials and in pharmaceuticals. Moreover, this method could be also extended for estimation of these drugs in biological fluids after a suitable clean-up procedure.

\section{References}

[1] El-Walily A.F., El-Yazbi F.A., Belal S.F., Abdel-Razak O. (1997), Anal. Lett., 30 (11): 2029.

[2] Rabouan S.G., Sourtois P., Barthes D. (1996), Farmaco, 51: 739.

[3] Morros A., Borja L., Segura J. (1985), J. Pharm. Biomed. Anal., 3 (2): 149.

[4] Kanda T., Ohtsu Y. (1998), Chromatography, 19 (4): 332.

[5] Fukushima T., Kato M., Santa T., Imai K. (1995), Analyst, 120 (2): 381.

[6] Kwok D.W., Igwemezie L., Kerr C.R., McErlane K.M. (1994), J. Chromatogr. B. Biomed. Appl., 661 (2): 271.

[7] Tateishi T., Harada K., Ebihara A. (1994), J. Liq. Chromatogr., 17 (3): 659.

[8] Abu Nassif M.A. (1991), Farmaco, 46 (3): 501.

[9] Stefan R.I., Ionescu M.S. (1995), Anal. Lett., 28 (6): 991.

[10] Proksa B., Proksova A. (2000), Pharmazie, 55 (5): 393.

[11] Kang J.W., Ou Q.Y. (1998), J. Chromatogr. A, 795 (2): 394.

[12] United States Pharmacopoeia (2000), 24, NF 19, Asian Edition, p. 1109.

[13] British Pharmacopoeia (1998), HM Stationary Office, London, p. 896 \& 1816.

[14] Moffat A.C., Jackson J.V., Moss M.S., Widdop B., Greenfield E.S. (1986), Clarke's Isolation and Identification of Drugs, Pharmaceutical Press, London, p. 782.

[15] United States Pharmacopoeia (2000), 24, NF 19, p. 2231 \& British Pharmacopoeia (1998), Vol. II, Appendix ID, A 101. 
[16] Buuren C.V., Lawrence J.F., Brinkman U.A., Honigberg I.L., Frei R.W. (1980), Anal. Chem., 52: 700.

[17] Derendorf H., Garrett E.R. (1983), J. Pharm. Sci., 72 (6): 630.

[18] Fernandez P., Alder A.C., Suter M.J., Giger W. (1996), Anal. Chem., 68 (5): 921.

[19] Kuemmerer K., Eitel A., Braun U., Hubner P., Daschner F., Mascart G., Milandri M., Reinthaler F., Verhoef J. (1997), J. Chromatogr. A, 774 (1-2): 281.

[20] Roach A., McCormick D. (1987), Measurement, Statistics and Computation, 2-nd Edition, Wiley, New York, p. 348.

[21] Miller J.C., Miller J.N. (1993), Statistics for Analytical Chemistry, Ellis Horwood, Chichester, 3rd Edition. 